\title{
Thimble-Chamber Calibration on Soft X-rays
}

\author{
By Frank H. Day
}

\begin{abstract}
Condenser $r$-meter thimble chambers of various types have been calibrated against a standard free-air ionization chamber to indicate the wavelength dependance of their readings after exposure to X-rays excited by potentials ranging from 10 to 200 kilovolts. X-ray tubes with low inherent filtration are used. Calibrations are conducted for minimumfilter conditions, and the effect of added filtration on the calibrations is also noted. It has been observed, for instance, that a chamber that reads correctly for filtered radiation of 100 -kilovolt quality is in error by a factor of 2.3 for 10 -kilovolt $\mathrm{X}$-rays.
\end{abstract}

\section{Introduction}

Thimble chambers of the Victoreen type are normally designed for the measurement of relatively hard radiations and are customarily calibrated with X-ray beams that are produced by tubes with an applied voltage of 70 to $200 \mathrm{kv}$, and which are subjected to some preliminary filtration, at least $2 \mathrm{~mm}$ of aluminum or $0.2 \mathrm{~mm}$ of copper. Over this range, the chambers yield readings that are practically independent of the radiation quality when calibrated against a free-air chamber. However, in the absence of any other ionization chamber, they are frequently used for measuring very much softer radiations than those for which they are designed or calibrated. Numerous cases have come to our attention where the red Bakelite 25- $r$ chamber has been employed to measure radiations produced by potentials as low as 20 or $30 \mathrm{kv}$, and it has been obvious in many cases that the users have had no reasonable idea as to the magnitude of the errors ${ }^{1}$ involved. It therefore appeared to be desirable to investigate the magnitude of these errors and provide some quantitative data thereon.

Measurements cited in this paper with respect to the effect of added filtration on the calibration relate primarily to one $25-r$ chamber. The effect of thimble-chamber type is studied with red Bakelite 25-r, 100-r, and 250-r chambers, as well as the newly designed $250 r$ nylon chamber. When calibrations are made in a given $\mathrm{X}$-ray beam, or in different beams of exactly the same quality, then,

\footnotetext{
${ }^{1} \mathrm{By}$ "error" is meant the departure of $r$-meter readings from those of a standard free-air chamber.
}

as noted below, different chambers of the same type generally require the same corrections. $^{2}$ However, chambers of different types require different corrections.

Although the results reported here may be taken as typical, the very magnitude of the corrections required indicates that whenever a thimble chamber is to be used for precise measurement in the very soft $\mathrm{X}$-ray region, it should be specifically calibrated for the exact conditions under which it will be used. This becomes more important, the lower the X-ray excitation potential. The wave form of the excitation potential becomes increasingly more important at the lower potentials also.

It is desirable for thimble-chamber readings to bear a constant relationship to those of a standard free-air chamber over a wide range of X-ray quality. To achieve this, it is necessary that the type of absorption and scattering in the thimble chamber wall be equivalent to that which is observed in air, and that the absorption in the wall thickness be negligible in comparison with the transmission through it, or that the secondary corpuscular radiation within the cavity be enhanced by means of a suitable internal coating of the wall to compensate for radiation lost by absorption in the wall thickness.

The practical attainment of this requirement is not difficult for 50 to $200-\mathrm{kv}$ X-rays emitted by a tube whose inherent filtration is at least $0.2 \mathrm{~mm}$ of copper. For example, in the measurement of

\footnotetext{
2 "Correction," as here used, is a factor by which $r$-meter readings must be multiplied to make them agree with those of a standard free-air chamber.
} 
radiation from such a tube, the Victoreen 25- $r$ thimble chamber, with its $0.6-\mathrm{mm}$ red bakelite wall, reads only 5 percent low for $50-\mathrm{kv}$ radiation if it reads correctly for 100 to $200-\mathrm{kv}$ radiation.

The problem is much more difficult, however, in the case of long-wavelength X-rays, produced by a tube whose inherent filtration is, say, $1.5 \mathrm{~mm}$ of beryllium. X-rays as long as $4 \mathrm{~A}$ in wavelength are available in measureable quantity from such a tube, and these are readily absorbed, even in a few centimeters of air. There are applications where it is necessary to determine the exposure at or near the portal of a beryllium-window tube. A properly designed chamber, therefore, should measure 4-A X-rays, (approximately 3-kv quality) with a minimum of correction.

An attempt has been made in this direction, ${ }^{3}$ utilizing an ionization chamber having a beryllium wall. The use of an open-air chamber has been reported, ${ }^{4}$ utilizing a limiting diaphragm and a mesh-type electrode system consisting of silk threads soaked in India ink to make them electrically conducting. A similar chamber has also been constructed, ${ }^{5}$ substituting, in place of the mesh of silk threads, Kodapak I, which is cellulose acetate sheet 0.00088 in. thick containing some plasticizer. Its calibration at the National Bureau of Standards yields scale factors (roentgens per scale division), which vary by less than 8 percent as the X-ray excitation potential is varied from 7.5 to $90 \mathrm{kv}$ and the filtration from the minimum of $1.5 \mathrm{~mm}$ of beryllium plus $100 \mathrm{~cm}$ of air to this plus $4 \mathrm{~mm}$ of aluminum.

\section{Experimental Procedure and Results}

In this study, the usual substitution method was employed in the calibration of the thimble chambers, the radiation being first measured with a standard guarded-field, free-air ionization chamber and then with the thimble chambers placed in the position of the limiting diaphragm. The filtration between the X-ray tube portal and the ionization chamber is, in all instances, $100 \mathrm{~cm}$ of air at approximately $760 \mathrm{~mm} \mathrm{Hg}$ pressure and $22^{\circ} \mathrm{C}$. It is necessarily a part of the minimum filtration, although it is not hereinafter so designated.

\footnotetext{
3 J. A. Victoreen, Z. J. Atlee, and E. D. Trout, Am. J. Roent. 53, 391 (1945).

${ }^{4}$ Edith H. Quimby and Elizabeth F. Focht, Am. J. Roent. 50, 653 (1943).

${ }^{5}$ Personal communication, H. E. Seemann (Eastman Kodak Co., Rochester,
}

The standard ionization chamber used in this comparison was the one designed for measuring radiations produced by potentials up to $200 \mathrm{kv}$. It has an air path of $21.1 \mathrm{~cm}$ between the limiting diaphragm and the front edge of the collector plate. ${ }^{6}$ Corrections required for the air absorption over this distance vary from about 0.5 percent for $200-\mathrm{kv}$ radiation to 33 percent for $10-\mathrm{kv}$ radiation, ${ }^{7}$ and these have been applied in this calibration.

"Constant" ${ }^{8}$ potentials, varied in steps between 10 and $200 \mathrm{kv}$, were employed on each of two tungsten-target X-ray tubes used in the calibration. The first was a thin-walled $(1.3 \mathrm{~mm})$ cerium-glass tube, which offers about as little inherent filtration as any glass-window tube. The second was a $200-\mathrm{kv}$ water-cooled tube whose window thickness is only $1.5 \mathrm{~mm}$ of beryllium, and which has almost no inherent filtration.

The $r$-meter readings were observed over approximately the same scale range to avoid any possible scale-factor error and were readily reproduced within \pm 1 percent. This is an over-all error, which includes fluctuations in the calibrating equipment of not greater than \pm 0.3 percent.

Figure 1 shows thimble-chamber corrections using the beryllium-window tube over the range of 10 to $100 \mathrm{kv}$, and for filtrations ranging from the minimum of $1.5 \mathrm{~mm}$ of beryllium to this plus $6 \mathrm{~mm}$ of aluminum of added filtration. For example, it is observed for the minimum filtration, that the correction is 1.52 at $100 \mathrm{kv}$ and increases to 2.3 at $10 \mathrm{kv}$. It is obvious that when such a large correction is necessary, the calibration of a thimble chamber for use with very soft radiations must be made with considerable care. In fact, some earlier studies ${ }^{9}$ indicate the necessity of effecting a special calibration for each specific tube in conjunction with its own specific potential source. The insertion of as little as $1 \mathrm{~mm}$ of aluminum in the beam, however, reduces the error from 52 to 3 percent at $100 \mathrm{kv}$ and from 60 to 8 percent at $50 \mathrm{kv}$.

\footnotetext{
${ }^{6}$ Lauriston S. Taylor and George Singer, BS J. Research 5, 507 (1930) RP211.

7 Frank H. Day and Lauriston S. Taylor, J. Research NBS 40, 393 (1948) RP1883.

8 The power supply has a resistance-capacitance filter to reduce the ripple to 0.05 percent per milliampere current drain, and currents used in these calibrations did not exceed $10 \mathrm{ma}$.

${ }^{9}$ L. S. Taylor, G. Singer, and C. F. Stoneburner, BS J. Research 11, 293 (1933) RP592.
} 
Similar corrections using the 1.3 -mm glass-wall tube are shown in figure 2. Here, for minimum filtration, it is seen that the correction varies from 1.17 at $100 \mathrm{kv}$ to 1.34 at $20 \mathrm{kv}$. Comparing these corrections with those of the $0.3-\mathrm{mm}$ aluminum curve in figure 1, it is found that they are closely alike over the whole potential range, indicating that the $1.3-\mathrm{mm}$ of cerium glass is equivalent in absorption to approximately $0.3-\mathrm{mm}$ of aluminum.

Calibration curves for the beryllium-window tube and copper filtrations at potentials up to 200 $\mathrm{kv}$ are shown in figure 3 . It is observed that until approximately $0.22-\mathrm{mm}$ of copper filtration is added to the beam, the errors at low potentials

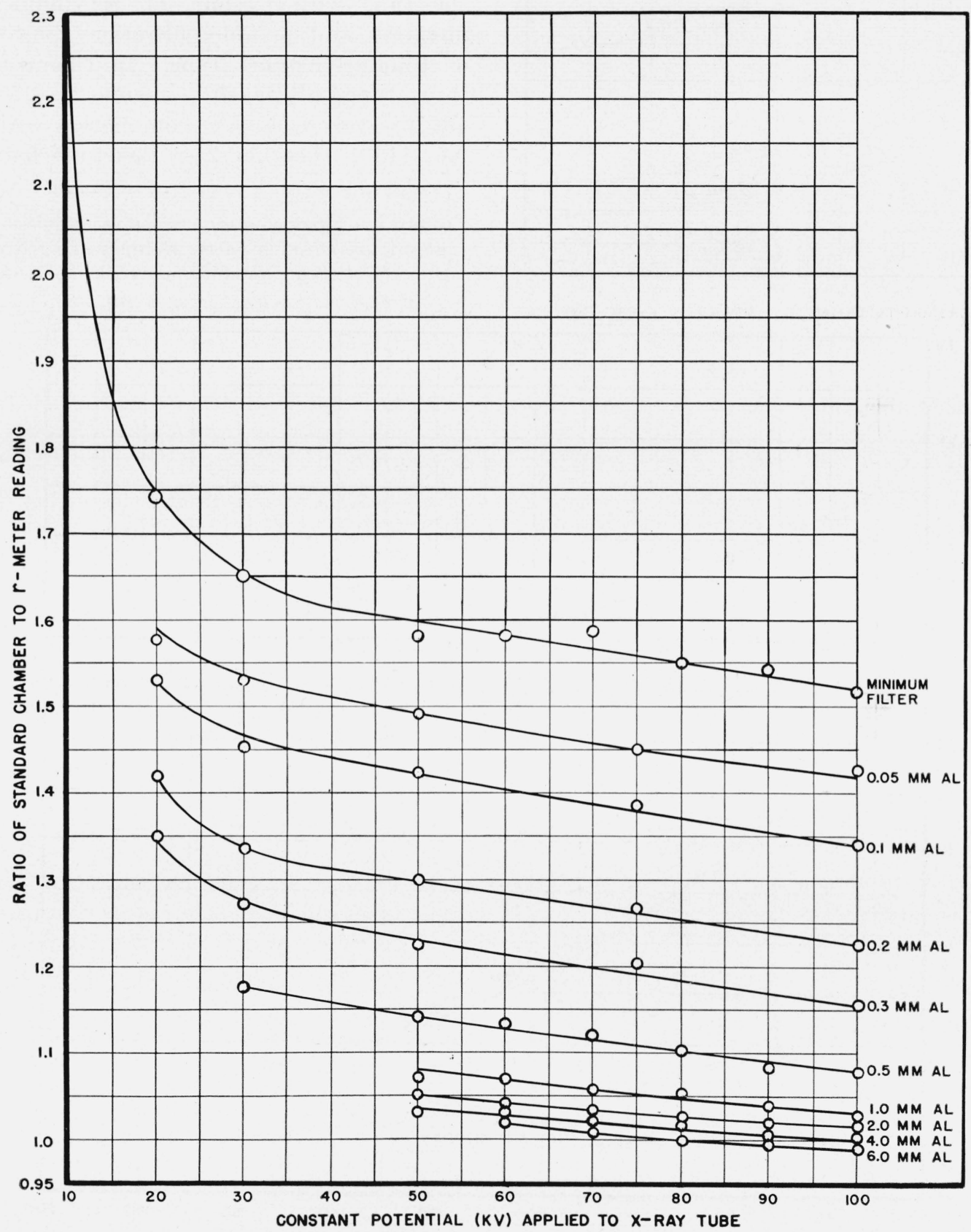

FIGURE 1. Effect of X-ray excitation potential and added aluminum filtration on the readings of $r$-meter No. 1193 and $25-r$ Bakelite thimble chamber, using X-ray tube with $1.5 \mathrm{~mm}$ of beryllium inherent filtration. 


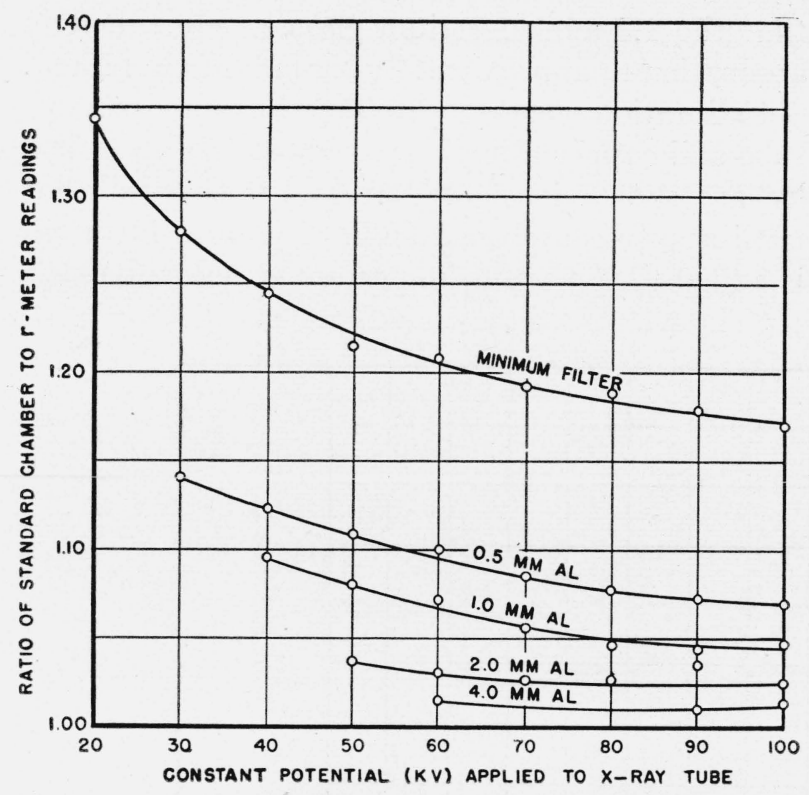

are relatively large, 11 percent, for example, at $50 \mathrm{kv}$ and $0.11-\mathrm{mm}$ of copper filtration. However, for $0.22-\mathrm{mm}$ of copper filtration, the thimble chamber agrees with the standard within \pm 2 percent over the range of 50 to $200 \mathrm{kv}$, and additional filtration does not change the calibration curves observably.

In figure 4, calibration curves, for X-rays from the tube with $1.5-\mathrm{mm}$ of beryllium inherent filtration, and no added filtration, show the effect of thimble-chamber type. In this comparison, three types of Bakelite chambers $(250-r, 100-r$ and 25-r) were used in conjunction with $r$-meter No. 1193. Another 25-r Bakelite chamber was

FIGURE 2. Effect of X-ray excitation potential and added aluminum filtration on the readings of r-meter No. 1193 and 25-r Bakelite thimble chamber, using X-ray tube with 1.3- $\mathrm{mm}$ of cerium glass inherent filtration.

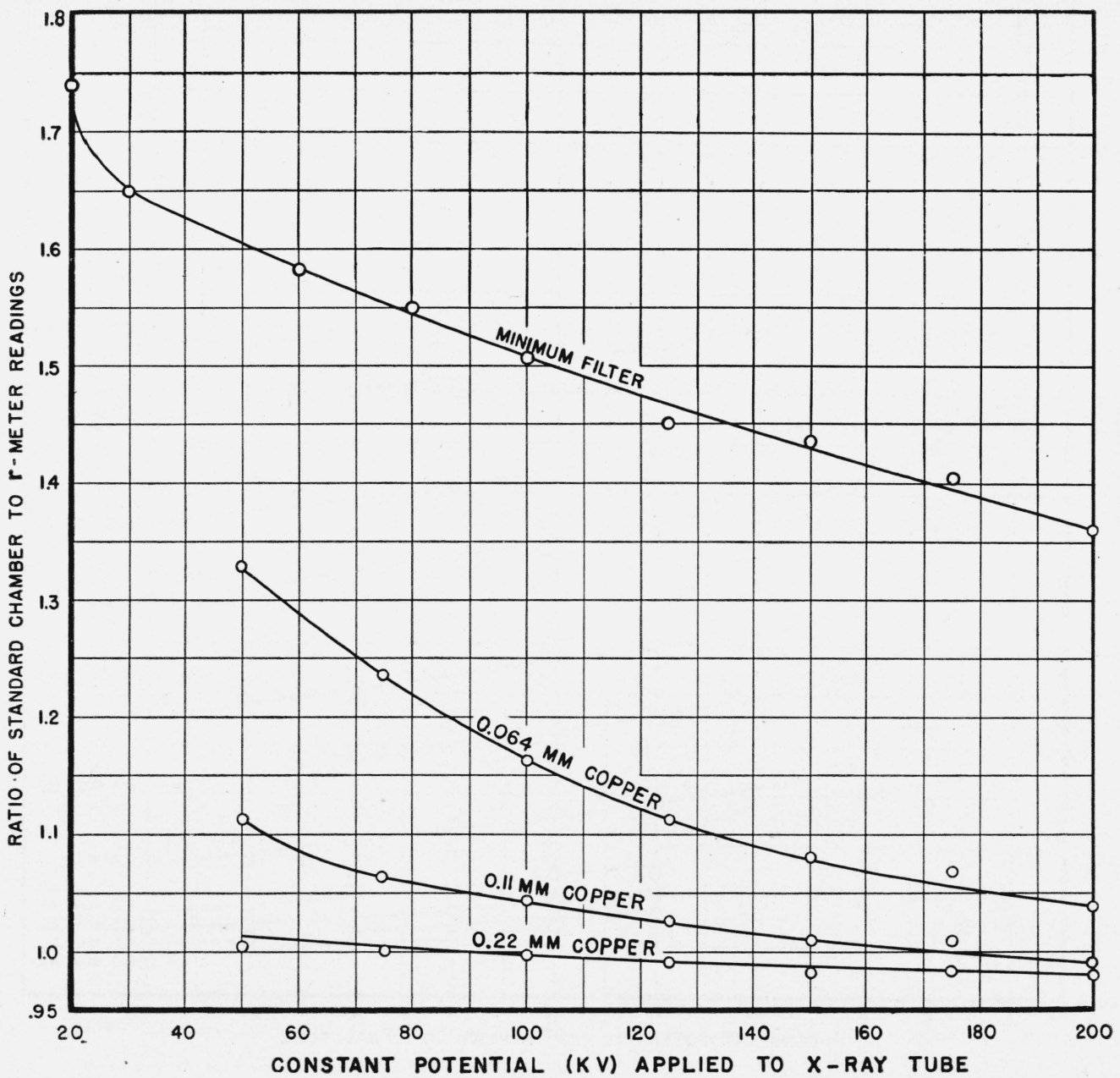

FIgURE 3. Effect of X-ray excitation potential and added copper filtration on the readings of r-meter No. 1193 and 25-r Bakelite thimble chamber, using X-ray tube with 1.5- $\mathrm{mm}$ of beryllium inherent filtration. 


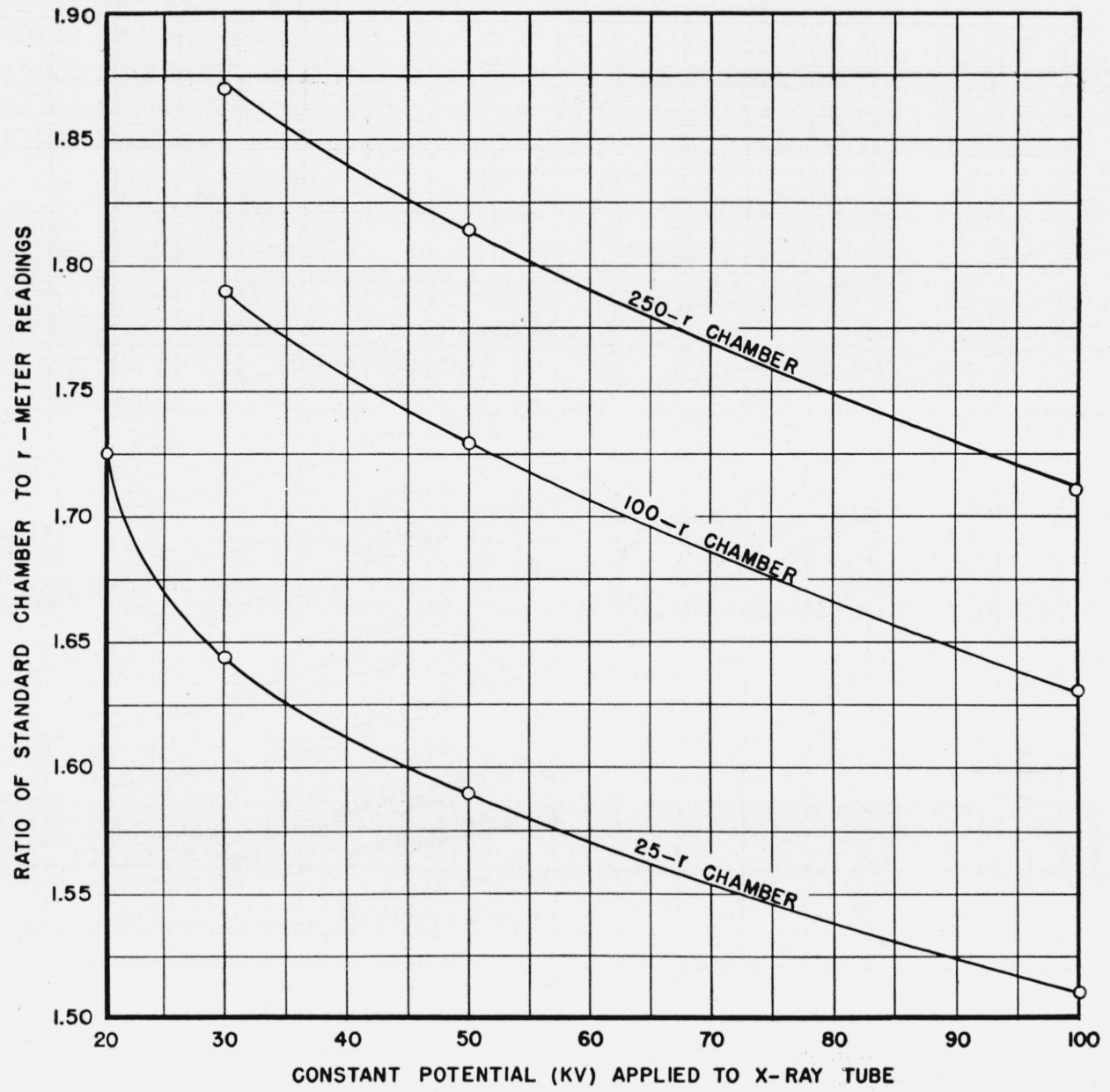

FIGURE 4. Effect of X-ray excitation potential on the readings of various types of Bakelite thimble chambers, using X-ray tube with 1.5-mm of beryllium inherent filtration and no added filtration.

The 250- $r$, 100- $r$, and 25- $r$ chambers are each used in conjunction with $r$-meter No. 1193. Another 25- $r$ chamber used in conjunction with $r$-meter No. 1282, yields readings that are the same within experimental error, as those observed with the 25-r chamber, which were read on $r$-meter No. 1193 .

used in conjunction with $r$-meter No. 1282. The readings of the two $25-r$ chambers and r-meters are the same, within experimental error, whereas those of the 100- $r$ and 250- $r$ chambers show substantially greater departure from those of the standard chamber. It is interesting to note that the calibration curves for these chambers run closely parallel to each other. For example, the correction factor to the $100-r$ and 250- $r$ chambers are 1.63 and 1.71 , respectively, at $100-\mathrm{kv}$, and 1.79 and 1.87 , respectively, at $30-\mathrm{kv}$, or differences of 0.08 at each potential.

A 250-r nylon chamber with a 0.005-in. wall thickness has recently become commercially available, and its calibration is shown in figure 5 using an X-ray tube with $1.5 \mathrm{~mm}$ of beryllium inherent filtration. For minimum-filtered radiation, this chamber reads in error by 31.5 to 28 percent over the range of 30 to $100 \mathrm{kv}$. This is in contrast with an error in the 250-r red bakelite chamber readings of 87 to 71 percent in the same excitation potential range, as seen in figure 4 .

Table 1 shows the aluminum half-value layers for 10 to $200-\mathrm{kv}$ X-rays with filtration ranging from $1.5 \mathrm{~mm}$ of berryllium to this plus $6 \mathrm{~mm}$ of aluminum. For minimum filter the very small half-value layers, even at $200 \mathrm{kv}$, indicate that the great bulk of the absorbed radiation is extremely soft, perhaps in the region of 20 to $30 \mathrm{kv}$, in terms of the usual measuring techniques. As an example of the effect of X-ray beam quality upon thimblechamber calibration, it is observed in figure 5 that $6 \mathrm{~mm}$ of aluminum are required to reduce the correction factor to a minimum at $150 \mathrm{kv}$. Table 


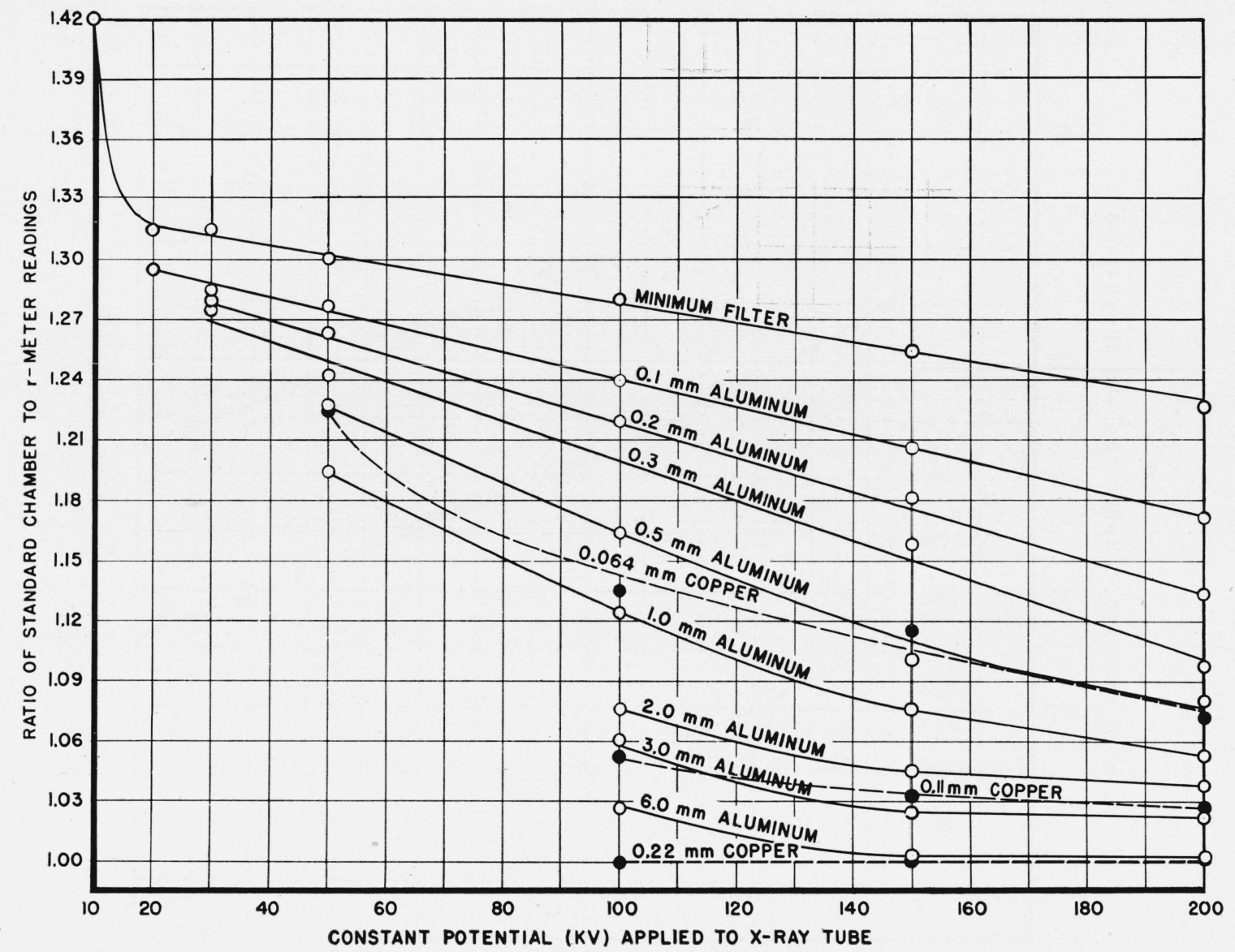

FIGURE 5. Effect of X-ray excitation potential and added filtration on the readings of r-meter No. 2449 and $250-r$ nylon thimble chamber, using x-ray tube with $1.5 \cdot \mathrm{mm}$ of beryllium inherent filtration.

1 indicates that a $150-\mathrm{kv}$ beam, prefiltered by $6 \mathrm{~mm}$ of aluminum, has a half-value layer of $7.6 \mathrm{~mm}$ of aluminum. This is in contrast with half-value layers ranging from 0.04 to $0.18 \mathrm{~mm}$ of aluminum

TABLE 1. Aluminum half-value layer determined with free-air ionization chamber as a function of filtration and of X-ray excitation potential

\begin{tabular}{|c|c|c|c|c|c|c|c|c|c|}
\hline \multirow{3}{*}{$\begin{array}{l}\text { Constant } \\
\text { potential }\end{array}$} & \multicolumn{9}{|c|}{ Half-value layer of aluminum } \\
\hline & \multirow{2}{*}{$\begin{array}{c}\text { Mini- } \\
\text { mum } \\
\text { filter } 1\end{array}$} & \multicolumn{8}{|c|}{ Added filter } \\
\hline & & $\underset{\mathrm{Al}}{0.1}$ & $\underset{\mathrm{Al}}{0.2}$ & $\underset{\mathrm{Al}}{0.3}$ & $\begin{array}{c}0.5 \\
\mathrm{~mm} \\
\mathrm{Al}\end{array}$ & $\begin{array}{c}1.0 \\
\mathrm{~mm} \\
\mathrm{Al}\end{array}$ & $\underset{\mathrm{Al}}{2.0}$ & $\begin{array}{c}3.0 \\
\mathrm{~mm} \\
\mathrm{Al}\end{array}$ & $\begin{array}{c}6.0 \\
\mathrm{~mm} \\
\mathrm{Al}\end{array}$ \\
\hline $10 \quad k v$ & $\begin{array}{l}m m \\
0.04\end{array}$ & $\begin{array}{l}m m \\
0.06\end{array}$ & $\begin{array}{l}m m \\
0.10\end{array}$ & $m m$ & $m m$ & $m m$ & $m m$ & $m m$ & $m m$ \\
\hline $20 \ldots \ldots$ & .08 & .11 & .15 & 0.18 & 0.22 & & & & \\
\hline $30 \ldots$ & .09 & .14 & .19 & .27 & .35 & 0.6 & 0.9 & 1.2 & \\
\hline $50 \ldots$ & .10 & .15 & .29 & .34 & .57 & 1.0 & 1.4 & 1.9 & 2.8 \\
\hline 100 & .11 & .19 & .37 & .48 & 1.0 & 1.8 & 2.9 & 3.6 & 5. 3 \\
\hline $150 \ldots$ & .14 & .29 & .64 & 1.2 & 2.0 & 3.2 & 4.6 & 5. 5 & 7.6 \\
\hline 200 & .18 & .40 & 1.3 & 2.1 & 3.4 & 4. 6 & 6.3 & 7. 1 & 8.8 \\
\hline
\end{tabular}

$11.5 \mathrm{~mm}$ of beryllium, plus $100 \mathrm{~cm}$ of air. at 10 and $200 \mathrm{kv}$, respectively, for minimum-filtered beams. In view of the large percentages of soft components in beams from beryllium-window tubes, it is to be expected that chambers used for measuring radiations from such tube, with no added filtration will be very critical as to design characteristics.

\section{Conclusion}

Thimble chambers can be calibrated for use with the very low-potential, lightly filtered radiations, such as produced by beryllium-window X-ray tubes. However, the errors may be as large as 130 percent for radiation produced at 10-kv constant potential.

Considerable care must be exercised in determining the amount and kind of filtration and potential employed in thimble-chamber calibrations for soft X-rays. These factors are relatively unimportant, however, where filtrations above $0.2 \mathrm{~mm}$ of copper or about $6 \mathrm{~mm}$ of aluminum are used.

Washington, January 26, 1948. 